\title{
Boundary Formation and Compartition in the Avian Diencephalon
}

\author{
Camilla W. Larsen, Lori M. Zeltser, and Andrew Lumsden \\ Medical Research Council Centre for Developmental Neurobiology, King's College London, London SE1 1UL, England
}

The diencephalon comprises three functionally distinct regions: synencephalon, dorsal thalamus, and ventral thalamus. Patterning of the diencephalon has been proposed to involve subdivision of its anteroposterior axis into segments, neuromeres or prosomeres (Bergquist and Kallen, 1954; Vaage, 1969; Figdor and Stern, 1993; Rubenstein et al., 1994; Redies et al., 2000; Yoon et al., 2000). However, the number and sequence of diencephalic neuromeres, or even their existence, are uncertain. We have examined the proposed subdivisions by morphology, gene expression, acquisition of boundary-specific phenotypes, and cell lineage restriction. We find that at stage 16 in chick the diencephalon is divided into synencephalon and parencephalon. The synencephalon exhibits neuromeric morphology, expresses Prox, and acquires neuromere boundary properties at its interface with both the midbrain and the parencephalon. Although the mesencephalic/synencephalic boundary restricts cell mixing, the synencephalic/parencephalic boundary does not. Similarly, there is no lineage restriction between the parencephalon and the more rostral forebrain (secondary prosencephalon). Subdivision of the parencephalon into ventral and dorsal thalamus involves the formation of a narrow intraparencephalic territory, the zona limitans intrathalamica (zli). This is correlated with the acquisition of cell lineage restriction at both anterior and posterior borders of the zli, the appearance of boundary-specific properties, and Gbx2 and DIx2 expression in dorsal thalamic and ventral thalamic territories, respectively. At stage 22, the synencephalon is divided into two domains, distinguished by differential gene expression and tissue morphology, but associated with neither a boundary phenotype nor cell lineage restriction. Our results suggest that the diencephalon does not have an overt segmental pattern.

Key words: diencephalon; CNS; segmentation; neuromeres; boundaries; compartments
The most studied example of segmentation in the vertebrate CNS is the hindbrain, the anteroposterior axis of which is subdivided into eight rhombomeres (Lumsden and Keynes, 1989; Cambronero and Puelles, 2000). Rhombomeres are units of cell lineage restriction (Fraser et al., 1990), arranged in an alternating repeat pattern of odd and even character, that are thought to be of central importance in the acquisition of subregional identity (Lumsden and Keynes, 1989; Lumsden and Krumlauf, 1996). The establishment of lineage restriction at the interfaces between rhombomeres, thereby containing cells within a developmental compartment, appears to be a function of immiscibility between odd and even cell populations (Wizenmann and Lumsden, 1997). The molecular basis of immiscibility has been ascribed to Eph/ ephrin interaction at the odd/even interface (Mellitzer et al., 1999; Xu et al., 1999). Boundary cells, which specialize after the formation of immiscibility interfaces, have characteristics that distinguish them from cells within the rhombomere bodies: interkinetic nuclear migration is disrupted (Guthrie et al., 1991), followed by an increase in extracellular space, expression of chondroitin sulfate proteoglycan (CSPG), laminin, weakly poly-

Received Dec. 27, 2000; revised Feb. 26, 2001; accepted March 14, 2001.

This work was supported by grants from the Wellcome Trust, the Medical Research Council, and the European Union. We thank E. Pollerberg for the gift of antibodies.

Correspondence should be addressed to Andrew Lumsden, MRC Centre for Developmental Neurobiology, King's College London, London SE1 1UL, England. E-mail: andrew.lumsden@kcl.ac.uk.

C. Larsen's present address: Division of Mammalian Development, National Institute for Medical Research, The Ridgeway, Mill Hill, London, NW7 1AA, England.

L. Zeltser's present address: Department of Genetics and Development, and Center for Neurobiology and Behavior, Columbia University, New York, New York 10032.

Copyright (C) 2001 Society for Neuroscience $0270-6474 / 01 / 214699-13 \$ 15.00 / 0$ sialylated NCAM, peanut agglutinin-binding proteins, and vimentin (Lumsden and Keynes, 1989; Layer and Alber, 1990; Heyman et al., 1995). Some or all of these specializations may reinforce the initial lineage restriction; some may also encourage the growth of axons and the formation of a precocious marginal zone, a further feature of maturing inter-rhombomere boundaries (Lumsden and Keynes, 1989).

Many attempts have been made to describe forebrain development in the context of neuromery. On the basis of morphology (Bergquist and Kallen, 1935; Coggeshall, 1964; Vaage, 1969; Keyser, 1972) and gene expression (Bulfone et al., 1993; Rubenstein et al., 1994), the diencephalon has been subdivided into three neuromeres (or prosomeres), corresponding with the synencephalon (presumptive pretectum), posterior parencephalon (presumptive dorsal thalamus), and anterior parencephalon (presumptive ventral thalamus). Figdor and Stern (1993) further divided the synencephalon into posterior and anterior regions and made the important claim that diencephalic neuromeres are, like rhombomeres, true neural segments, i.e., they are compartments defined by cell lineage restriction. Alternating expression patterns of acetylcholinesterase and PNA binding suggested a two-segment repeat, like the rhombomeres (Figdor and Stern, 1993).

Because it would be expected that the forebrain and hindbrain would share the same segmentation mechanism, we have attempted to characterize putative diencephalic neuromeres according to criteria established for rhombomeres. However, we find that of the proposed interneuromeric boundaries in the diencephalon, only those bordering the zona limitans intrathalamica (zli; a narrow stripe of cells that lies between the prospective dorsal and ventral thalami) and the mesencephalic/syn- 
encephalic $(\mathrm{m} / \mathrm{s})$ boundary exhibit both lineage restriction and boundary cell properties. Others, such as the synencephalic/ parencephalic (s/p) boundary, express boundary properties transiently, but there is no lineage restriction either here or between the diencephalon and the secondary prosencephalon [the anteriormost region of the neural tube that encompasses the preoptic area, hypothalamus, and telencephalon (Puelles et al., 1987)]. Finally, the intrasynencephalic boundary is not associated with either a boundary phenotype or cell lineage restriction. Our results therefore indicate that diencephalic patterning does not involve overt segmental subdivision.

\section{MATERIALS AND METHODS}

Chick embryos. Fertilized eggs (Rhode Island Red) were incubated at $37^{\circ} \mathrm{C}$ in $40-50 \%$ humidity, until the desired developmental stage was reached. Embryos for in situ hybridization and immunohistochemistry were fixed in $4 \%$ paraformaldehyde in PBS for $12 \mathrm{hr}$. For in vivo manipulation, eggs were windowed, and the embryo was visualized by sub-blastodermal injection of India ink.

Whole-mount immunohistochemistry was as described by Lumsden and Keynes (1989) with modifications: for the neurofilament-specific antibody RMO-270 (Zymed, San Francisco, CA), embryos were dehydrated through ascending methanol and incubated overnight at $-20^{\circ} \mathrm{C}$. The embryos were rehydrated and washed in PBS containing 1\% Tween (PBT). For all other antibodies used, embryos were immersed in cryoprotection solution (PBS containing 1\% Triton X-100, 5\% goat serum, and $8 \%$ sucrose) and incubated twice at $-20^{\circ} \mathrm{C}$ until just frozen, allowing the embryos to thaw and reach room temperature after each incubation.

Immunohistochemistry on sections was performed on both paraffin and frozen sections (standard protocols). Both types of section were bleached for $1 \mathrm{hr}$ in PBS containing $0.1 \% \mathrm{H}_{2} \mathrm{O}_{2}$ and blocked in $10 \%$ goat serum, $1 \%$ Triton in PBS for $2 \mathrm{hr}$. Sections were incubated overnight with the appropriate concentration of primary antibody, followed by six washes in PBS. Sections were incubated with a 1:100 dilution of peroxidase-conjugated secondary antibody for $4 \mathrm{hr}$ and developed as for whole-mount immunohistochemistry.

Antibodies. RMO-270 antibody (Zymed) was used at a 1:10,000 dilution. Both 5A5 and M1-B4 ascites were obtained from the Hybridoma Bank and were used at a 1:100 and 1:150 dilution, respectively. Vimentin and CSPG (Sigma, St. Louis, MO) were both used at a 1:100 dilution. Anti-NrCAM and anti-neural cell adhesion molecule (NCAM) were kindly donated from E. Pollerberg and used at concentrations of 1:5,000 and 1:30,000, respectively. Commercially available secondary antibodies appropriately labeled with Cy3 or PO (Jackson ImmunoResearch Laboratories) were used to detect the primary antibodies.

Nissl staining. Embryos were fixed in Bouin's (0.9\% picric acid, 9\% formaldehyde) and wax sectioned. Sections were incubated in Nissl stain for $12 \mathrm{hr}$ at room temperature, washed in $\mathrm{H}_{2} \mathrm{O}$, and differentiated by rinsing in $70 \%$ alcohol containing $1 \mathrm{~N}$ acetic acid until the stain remained only in cell membranes.

In situ hybridization was performed as described by Grove et al. (1998).

Scanning electron microscopy. Specimens were fixed in $2.5 \%$ glutaraldehyde for $4 \mathrm{hr}$ at $4^{\circ} \mathrm{C}$ and then washed in sodium phosphate buffer containing $8.5 \%$ sucrose for $4 \mathrm{hr}$ to $2 \mathrm{~d}$ at $4^{\circ} \mathrm{C}$. They were post-fixed in $1 \%$ osmium tetroxide in Millonig's constant osmolarity phosphate buffer at $4^{\circ} \mathrm{C}$ for $90 \mathrm{~min}$, followed by dehydration through ascending acetone. Embryos were then critical-point dried in an Emscop CPD 750 criticalpoint dryer, mounted onto metal stubs with carbon-conductive paint, coated with a thin layer of gold using a sputter coater (model SC500), and viewed using a model S520 scanning electron microscope.

Transmission electron microscopy. Embryos were fixed in 3.5\% glutaraldehyde in $0.1 \mathrm{M}$ phosphate buffer, $\mathrm{pH} 7.3$, at $4^{\circ} \mathrm{C}$ for $4 \mathrm{hr}$ and osmicated in $1 \%$ aqueous $\mathrm{OsO}_{4}$ for $30 \mathrm{~min}$, followed by dehydration in ascending methanol, washing in propylene oxide, 1:1 propylene oxide, and Taab Epon resin, and embedding in Epon. Ultrathin horizontal sections $(70-90 \mathrm{~nm})$ through the forebrain were cut with a diamond knife and mounted on Formvar-coated meshed grids. Dried sections were stained with lead citrate and uranyl acetate and viewed in a Hitachi H7000 transmission electron microscope.

Bromodeoxyuridine labeling. In vivo prepared embryos were labeled for $30 \mathrm{~min}$, each with $10 \mu \mathrm{l}$ of a $15 \mathrm{mg} / \mathrm{ml}$ bromodeoxyuridine (BrdU) solution, and fixed for $12 \mathrm{hr}$ with $4 \%$ PFA. After fixation, embryos were taken through the in situ hybridization and immunohistochemistry to detect BrdU.

Dextran labeling. With a tungsten needle, a small hole was cut through the mesenchyme and epithelium either just above the eye on the right side of the embryo (facing upward) or in the most anterior dorsal part of the midbrain. This hole was large enough to allow a micropipette to pass through without touching any tissue. To label cells, a mixture of rhodamine- and biotin-labeled dextrans was injected by iontophoresis. Injection was confirmed by using a fluorescence microscope. Embryos were fixed after $48 \mathrm{hr}$ survival and taken through in situ hybridization as described above. To detect labeled cells after in situ hybridization, embryos were incubated overnight with a 1:250 concentration of POconjugated streptavidin and detected as for the immunohistochemistry protocol.

\section{RESULTS}

\section{Subdivision of the diencephalon on the basis of morphology and Nissl staining}

Neuromeric morphology was originally defined as external bulges of neuroepithelium delineated by grooves, which appear on the ventricular surface as troughs delineated by ridges (von Baer, 1828). We have used three techniques to assess morphological subdivision of the diencephalon into putative neuromeres: scanning electron microscopy, Nissl staining, and immunohistochemical analysis of neuronal distribution.

The first morphological subdivision of the diencephalon is apparent at Hamburger and Hamilton stage $(\mathrm{HH}) 16$, when the synencephalon adopts a typical neuromeric phenotype (Fig. 1A$C)$. Its borders with the midbrain and parencephalon are marked by ridges extending from the dorsal midline and ending above the ventral midline (Fig. 1A, arrowhead). Neurofilament staining (Fig. $1 D$ ) reveals that the pattern of neurogenesis differs between the synencephalon and the dorsal thalamus at this stage: neurons have differentiated in the dorsal and ventral regions of the synencephalon, whereas they are confined to the ventral parencephalon, where neurons of the interstitial nucleus of Cajal are among the first to differentiate. At HH 16, axonal projections are confined to their respective neuromere of origin, except for the medial longitudinal fasciculus, which projects caudally along the ventral margin of the basal plate (Fig. 1D).

At $\mathrm{HH}$ 19, the parencephalon is divided into the dorsal thalamus posteriorly and the ventral thalamus anteriorly by the zli. Unlike the borders of the synencephalic neuromere, the zli is formed from an initially broad domain, which gradually narrows from the most ventral part of the alar plate toward the dorsal midline to form a prominent ridge on the ventricular surface (Zeltser et al., 2001). The ridge of the zli is first detectable by scanning electron microscope at $\mathrm{HH} 19$ and extends at an angle of $\sim 45^{\circ}$ relative to the s/p boundary (Fig. $1 E, F$ ). By HH 26, when the ridge has reached its full extent, just short of the dorsal midline, the dorsal thalamus bulges into the ventricular lumen. Because of this change in morphology and the gradual extension of the zli, the dorsal thalamus never adopts a typical neuromeric phenotype of a trough delineated by ridges. The prospective ventral thalamus does not exhibit neuromeric morphology either, because of both the gradual extension of the zli at its posterior border and the absence of a ridge at its anterior border with the secondary prosencephalon.

Neurogenesis has advanced considerably by HH 19 with the formation of a mantle zone in the synencephalon and dorsal thalamus but not yet within the ventral thalamus (Fig. 1G). The entire synencephalic neuromere is filled with neurons and axons (Fig. $1 H$ ), which are more tightly packed dorsally. Neurons in the dorsal thalamus are sparse but evenly distributed, and their axons 

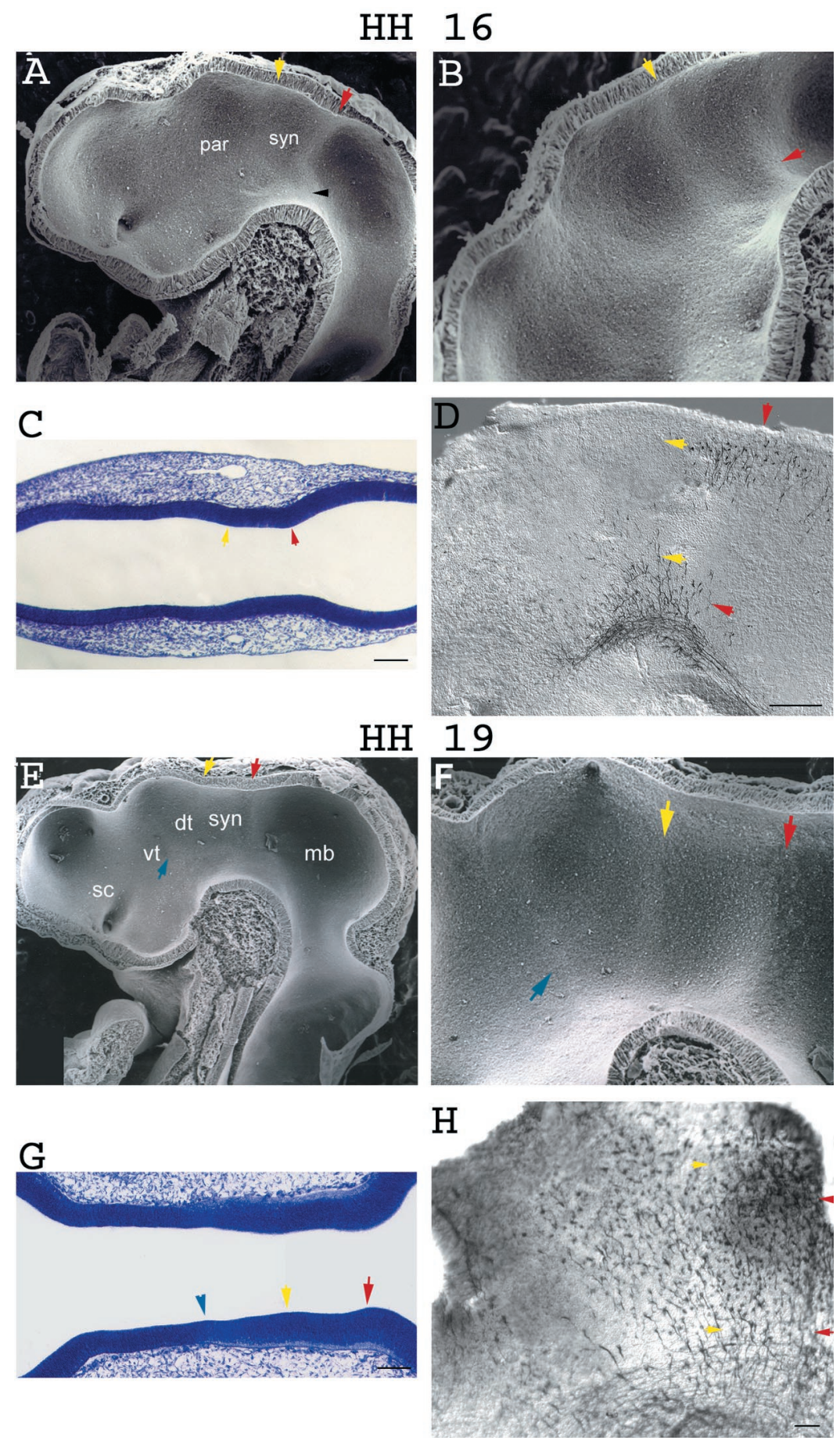

Figure 1. Early morphological subdivisions of the diencephalon. Anterior is to the left unless stated otherwise. Scale bars, $200 \mu \mathrm{m}$. The red arrowhead indicates the boundary between the midbrain and synencephalon $(\mathrm{m} / \mathrm{s})$. The yellow arrowhead indicates the boundary between the synencephalon and the parencephalon (s/p), and the blue arrowhead marks the intraparencephalic boundary (zli). The black arrowhead marks the basal plate at $\mathrm{HH} 16 . A-D, \mathrm{HH}$ 16. $E-H$, HH 19. $A, B, E, F$, Scanning electron micrographs of the ventricular surface of hemisected embryos at different angles to emphasize the ridges of the ventricular surface. $A$, $B$, Ridges colocalize with the $\mathrm{m} / \mathrm{s}$ and $\mathrm{s} / \mathrm{p}$ boundaries. $E, F$, The ridge that demarcates the zli begins to form (blue arrowhead). $E$, Medial view of embryo. $B$, Secondary prosencephalon anterior at the bottom. Magnifications: $A, 135 \times ; B$, $310 \times ; E, 110 \times ; F, 247 \times . C, G$, Horizontal Nissl-stained wax sections showing the morphological ridges at the ventricular surface. $D, H$, Whole-mount immunohistochemistry with the anti-neurofilament antibody RMO-270. The embryos have been hemisected and viewed from the pial side after the mesenchyme was removed. Both $D$ and $H$ show that neurons are more numerous in the synencephalon than in the rest of the diencephalon. syn, Synencephalon; par, parencephalon; $d t$, dorsal thalamus; $v t$, ventral thalamus; $m b$, midbrain; sc, secondary prosencephalon. project parallel to the nascent zli. In the ventral thalamus, neurons are predominantly localized ventrally and rostrally.

At $\mathrm{HH} 22$, the zli has thickened considerably at its ventral aspect, forming a prominent ridge (Fig. $2 A$ ). The ridge of the $\mathrm{s} / \mathrm{p}$ boundary is visible in dorsal and ventral positions (Fig. $2 B, C$, yellow arrowhead) but disappears in the intervening midlateral region. The anterior and posterior parts of the synencephalon adopt a different cellular morphology (Fig. 2A,B), corresponding to the subdivision noted by Figdor and Stern (1993). This change in morphology, however, is not associated with the formation of a ridge between the two synencephalic subunits, such as those observed at the zli and the $\mathrm{m} / \mathrm{s}$ and $\mathrm{s} / \mathrm{p}$ boundaries. Rather, the posterior part of the synencephalon bulges into the ventricular lumen to a greater extent than does the anterior part, which appears as a trough, and is delineated anteriorly by the $\mathrm{s} / \mathrm{p}$ boundary (Fig. $2 A-C$ ). The lack of overt neuromeric morphology indicates that any subdivision of the synencephalon does not involve the formation of an additional neuromere.

The pattern of neurogenesis and differentiation varies between the diencephalic areas. The thickness of the ventricular zone is uniform throughout the synencephalon, except in posterior regions, where the marginal zone appears to be broader compared with the anterior part (Fig. 2C). The posterior synencephalic neuronal population is wider at the dorsal aspect, narrowing 

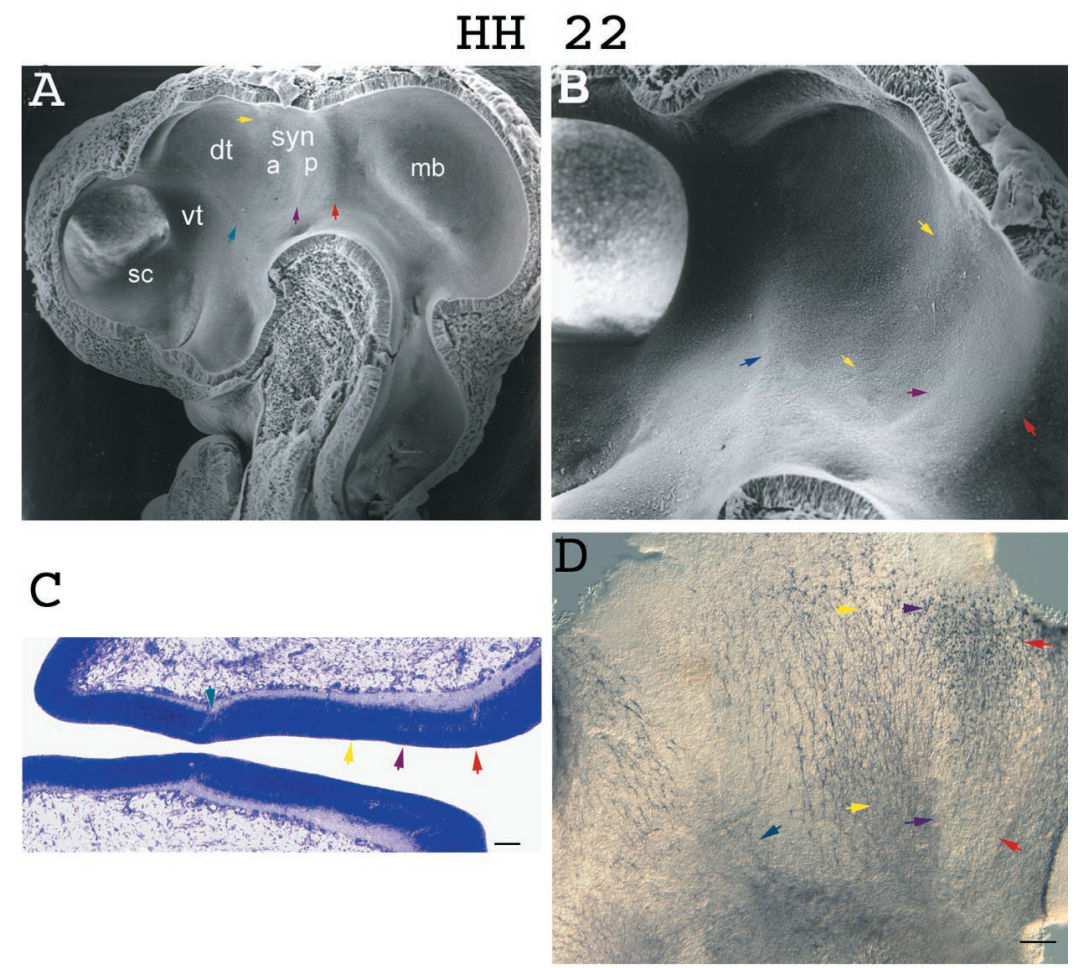

Figure 2. Later morphological subdivisions of the diencephalon. Anterior is to the left unless stated otherwise. Scale bars, $200 \mu \mathrm{m}$. The red arrowhead indicates the boundary between the midbrain and synencephalon $(\mathrm{m} / \mathrm{s})$. The purple arrowhead indicates the interface between the anterior and posterior synencephalon. The yellow arrowhead indicates the boundary between the synencephalon and the parencephalon $(\mathrm{s} / \mathrm{p})$, and the blue arrowhead marks the intraparencephalic boundary (zli). $A-D, \mathrm{HH} 22 . E-G$, HH E5. $A, B, E, F$, Scanning electron micrographs of the ventricular surface of hemisected embryos at different angles to emphasize the ridges in the ventricular surface. $A$ and $D$ show that ridges have formed along all putative diencephalic boundaries, except between the anterior and posterior part of the synencephalon. $E-G$, At this later stage the ventricular surface of the diencephalon appears as a series of large grooves and broad ridges. $A, E$, Lateral view. $B$, Picture taken from the midbrain, so that anterior is at the left and posterior is toward the bottom right-hand corner. Magnifications: $A, 50 \times$; $B, 100 \times ; E, 25 \times ; F, 75 \times . C, G$, Horizontal Nissl-stained sections showing the morphological ridges and differences in cell density. $D$, Whole-mount immunohistochemistry with RMO-270 showing the extent of neurogenesis within the different parts of the diencephalon. The embryo has been hemisected and is viewed from the pial side after the mesenchyme was removed. Abbreviations as for Figure 1.

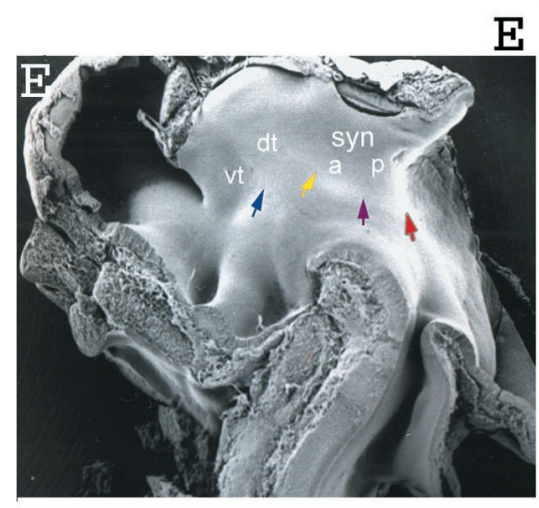

E 5

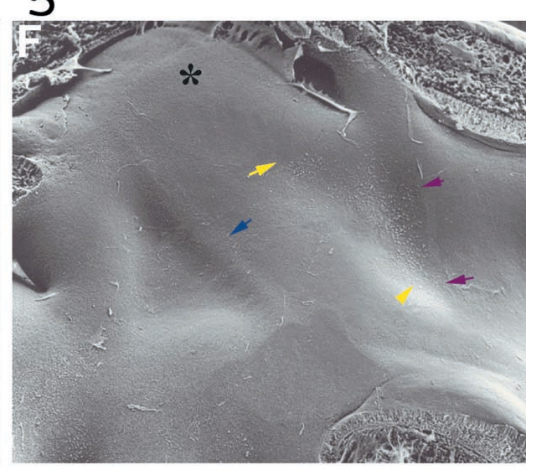

G

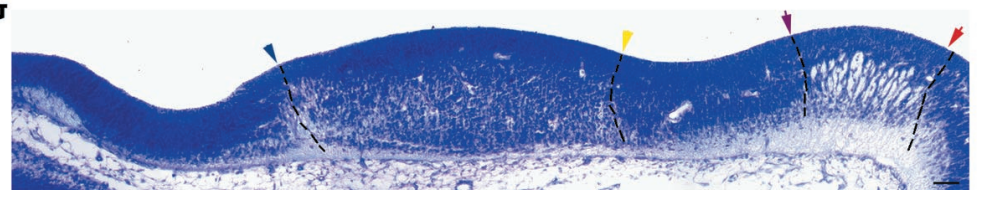

down toward the ventral part of the alar plate (Fig. 2D). The anterior synencephalic neuronal population, on the other hand, is denser at the ventral aspect of the neural tube. Unlike the neurons in the posterior part of the synencephalon, the anterior synencephalic neuronal population seems to project toward and across the boundary into the dorsal thalamus, indicating that the $\mathrm{s} / \mathrm{p}$ boundary is not a barrier to extending axons as suggested by its appearance at $\mathrm{HH}$ 16. The area around the zli still lacks neurofilament staining, although the ventral thalamus neuronal population has expanded caudally after $\mathrm{HH} 19$.

By embryonic day (E) 5, all the ridges apart from the zli have disappeared, and the ventricular surface now appears as a series of wide troughs and bulges (Fig. $2 E-G$ ). The posterior part of the synencephalon bulges considerably into the ventricular lumen, whereas the anterior part of the synencephalon is left as a large triangular groove. The dorsal thalamus appears as an elongated quadrant, increasing in width ventrally and narrower at the dorsal aspect. The angle of this quadrant corresponds to that of the zli, which is still $45^{\circ}$. Within the extreme dorsal part of the dorsal thalamus there is a rounded region (Fig. $2 F$, asterisk), which will form the epithalamus. Note also that the zli has now thickened throughout its entire length.

Neurogenesis has advanced considerably, and independent neuronal groups are no longer readily distinguishable (data not shown). However, differences between subregions of the diencephalon can be recognized in the Nissl preparations (Fig. 2G). The ventricular and mantle zones are distinct, where the former appears as a tightly packed, heavily stained band of cells along the ventricular surface. The mantle zone is packed less densely and is considerably thicker than the ventricular layer at this stage. The posterior commissure spans most of the mantle zone of the posterior synencephalon, visible as faint axonal staining and a few 
scattered stained cells. The cells within the anterior part of the synencephalon, on the other hand, are more tightly packed throughout most of the mantle zone. The zli is almost devoid of cells within the mantle region, whereas the ventricular zone is still evenly packed, although thinner than the rest of the diencephalon. Within the ventral thalamus, the cells are tightly packed, and it is difficult to distinguish mantle and ventricular zones. Although morphological criteria can be used to divide the diencephalon into four domains, only the synencephalon at $\mathrm{HH} 16$ has a neuromeric morphology.

\section{Subdivision of the diencephalon on the basis of gene expression}

In several developmental systems, gene expression domains demarcate patterning units or compartments (Lawrence and Struhl, 1996; Lumsden and Krumlauf, 1996). In the diencephalon, the spatially restricted expression of genes encoding several signaling molecules and transcription factors has been noted for the ventral thalamus and dorsal thalamus (Bulfone et al., 1993). However, molecular markers for the synencephalon have not been reported. Previous gene expression studies have been performed in diverse species and have concentrated on one or two stages of development. It remains unclear from these studies when expression is initiated within the individual domains. We have therefore performed a stage-by-stage in situ analysis of genes expressed within the avian diencephalon.

Prox, a homeobox gene homologous to Drosophila prospero (Oliver et al., 1993), is expressed exclusively within the synencephalon until at least E7, where its expression is initiated ventrally and expands dorsally as development proceeds. Expression is first detected at $\mathrm{HH} 16$ in a narrow band in the ventral synencephalon (Fig. $3 A$, red arrow). The onset of Prox expression thus correlates temporally and spatially with the physical appearance of the synencephalic neuromere. At $\mathrm{HH}$ 21, expression extends through the ventral part of the synencephalon and appears to decrease in a gradient toward the dorsal midline (Fig. $3 B$ ). After the intrasynencephalic subdivision at $\mathrm{HH} 22$, Prox expression is confined to the posterior synencephalon, and by E5, Prox is expressed exclusively within and demarcates the posterior synencephalon (Fig. 3C).

Similar to Prox, expression of the homeobox genes Gbx2 and Dlx2, which demarcate the dorsal thalamus and ventral thalamus, respectively (Bulfone et al., 1993), is initiated ventrally and expands dorsally as development proceeds. Gbx2 is first expressed at $\mathrm{HH} 19$ in the ventral aspect of this region (data not shown). At HH 21, Gbx2 expression has expanded caudally along the ventral aspect of the dorsal thalamus and dorsally along the posterior border of the zli (Fig. 3D). This dorsal and caudal extension continues (Fig. 3E) until E5, when it reaches its dorsal limit close to the midline (data not shown). Gbx2 is expressed primarily in the mantle zone. Although the boundary of expression between the dorsal thalamus and the synencephalon is sharply defined, some $G b \times 2$-positive cells are seen within the anterior synencephalon (Fig. 3F, black arrow).

Dlx2 is also expressed initially at HH 19 (data not shown) but in the ventral-most part of the ventral thalamus, adjacent to the zli. By $\mathrm{HH}$ 21, expression extends anteriorly and dorsally along the anterior border of the zli (Fig. 3G). There is also a line of expression from the ventral point of the Dlx2 domain extending into the ventral telencephalon. Over the next $2 \mathrm{~d}$ of development, Dlx2 expression spreads dorsally and anteriorly to fill the entire ventral thalamus by E5.5 (Fig. $3 H$ ). This progressive expansion appears to be further advanced just rostral to the zli. Thus, the expression of both Gbx2 and Dlx2 coincides with the developmental stage when the parencephalon becomes subdivided by the zli.

Although we did not identify any genes expressed exclusively within the anterior synencephalon, the expression level of Lunatic fringe ( $L-f n g$ ) is higher in the anterior than posterior part. L-fng is a member of the Fringe family of glycosyltransferases that modulate the Notch signaling pathway (Fleming et al., 1997; Panin et al., 1997; Klein and Arias, 1998; Bruckner et al., 2000; Moloney et al., 2000). L-fng exhibits a dynamic expression pattern in the diencephalon from early developmental stages, as has been reported previously (Zeltser et al., 2001). From HH 20 onward (Fig. 3I), its expression is downregulated within the posterior part of the synencephalon. Expression is absent from the extreme dorsal region of the posterior synencephalon but remains ventrally. The high levels of $L-f n g$ expression in the anterior synencephalon are continuous with the dorsal thalamus. At HH 23 (Fig. $3 J), L-f n g$ is downregulated within the caudal aspect of the dorsal thalamus, leaving a domain of strong expression demarcating the anterior synencephalon and a low level of expression within the posterior synencephalon. The difference in morphology between the posterior and anterior part of the synencephalon, visible from HH 22 (see above), thus correlates with the change in the expression levels of $L-f n g$ within the two subdivisions.

\section{Location of S-phase cells}

Segmentation and compartition in the hindbrain are associated with the generation of a unique boundary morphology (Lumsden and Krumlauf, 1996). Compared with the hindbrain, little is known about the boundary regions in the diencephalon. To characterize the diencephalic neuromere boundaries, and specifically to compare them with rhombomere boundaries, we examined the localization of S-phase cells by BrdU labeling and the expression of several cell adhesion and extracellular matrix molecules by immunohistochemistry.

S-phase nuclei are localized apically within rhombomere boundaries, as distinct from their juxta-basal location in rhombomere bodies (Guthrie et al., 1991). At HH 14, before subdivision in the diencephalon, S-phase nuclei are predominantly located basally within the ventricular zone, although more apically located nuclei can be found distributed randomly within the entire neuroepithelium (data not shown). At HH 16, with the s/p subdivision, S-phase nuclei are found apically on either side of the boundaries (Fig. 4A-C). This apical localization in the s/p boundary is transient and is lost by HH 18 (data not shown). At HH 21, $\mathrm{S}$-phase cells remain apically localized in the $\mathrm{m} / \mathrm{s}$ boundary and within the zli (Fig. 4D-F). Here, S-phase cells aggregate apically, predominantly within the rostral part of the ridge (Fig. $4 F$, white arrow), whereas a line with few S-phase cells is evident more caudally (Fig. $4 F$, open arrow). We noted another domain of apically localized S-phase cells (Fig. $4 F$, filled arrow) immediately posterior to the zli ridge. Throughout the rest of the diencephalon, S-phase cells are located basally, in the pial half of the neuroepithelium (Fig. 4D). At no time were S-phase cells seen apically at the putative intrasynencephalic boundary. Thus apically located S-phase cells are found as an enduring feature only at the $\mathrm{m} / \mathrm{s}$ boundary and in the zli.

\section{Expression of boundary markers}

We examined whether CSPG, tenascin, and vimentin, which are expressed preferentially within rhombomere boundaries, are sim- 

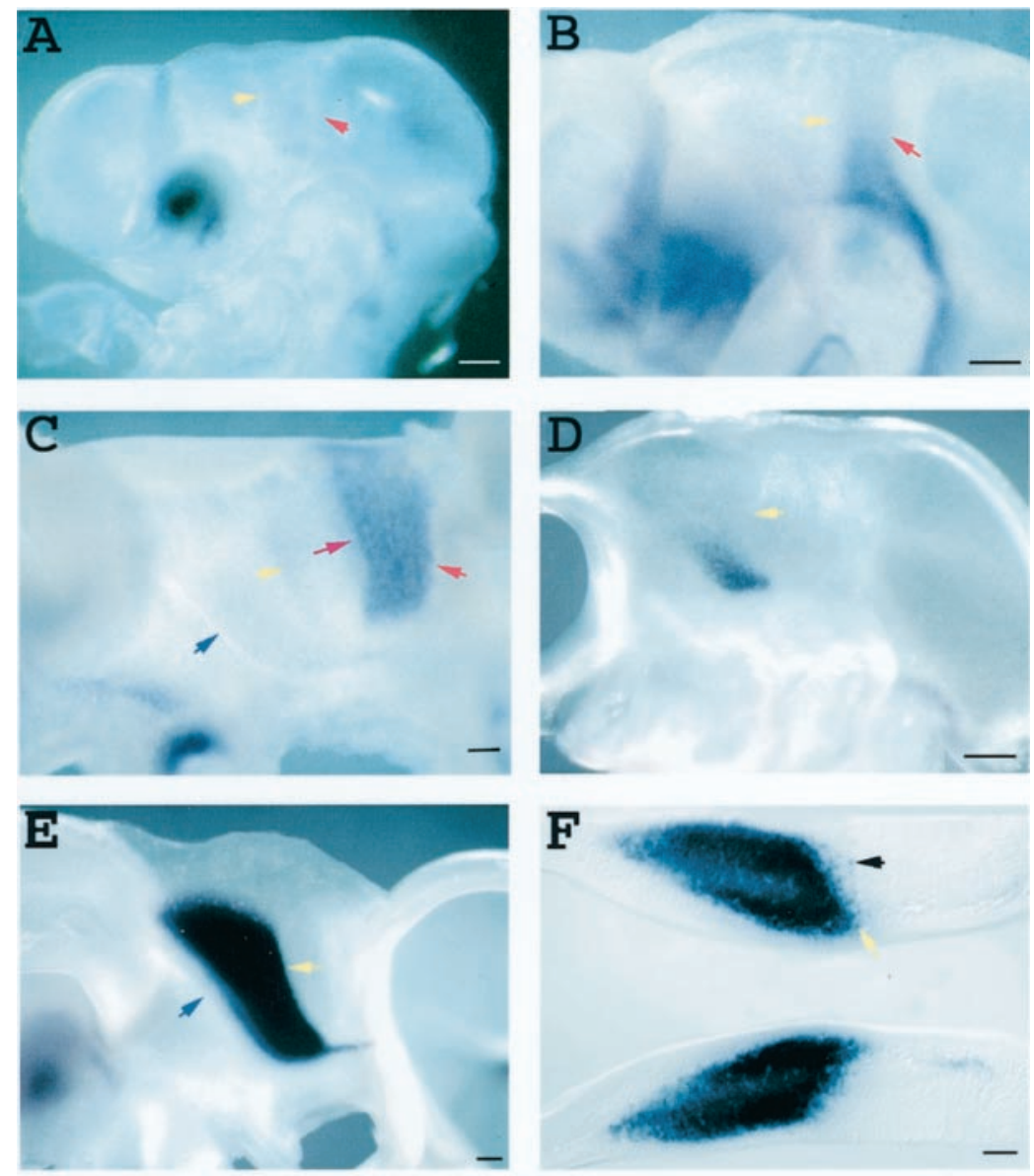
Anterior is to the left unless stated otherwise. Scale bars, 200 $\mu \mathrm{m}$. The red arrowhead indicates the boundary between the midbrain and synencephalon $(\mathrm{m} / \mathrm{s})$. The purple arrowhead indicates the interface between the anterior and posterior synencephalon. The yellow arrowhead indicates the boundary between the synencephalon and the parencephalon $(\mathrm{s} / \mathrm{p})$, and the blue arrowhead marks the intraparencephalic boundary (zli). All embryos are hemisected and viewed from the ventricular surface. Expression of each gene is initiated ventrally and spreads slowly dorsalward with development. $A-C$, Wholemount in situ hybridization (ISH) with a Prox probe, initially expressed in a small domain within the synencephalon and eventually demarcating the posterior synencephalon $(C) . A$, HH 16; $B, \mathrm{HH} 21$; and $C$, E5. $D, E$, Whole-mount ISH with a $G b x 2$ probe, showing the gradual dorsal spread of expression up to $\mathrm{HH} 26$ when the entire dorsal thalamus is $G b \times 2$ positive. $D$, HH 21; E, HH 26. F, A coronal section of a whole-mount $G b x 2$ ISH embryo, showing Gbx2-positive cells within the synencephalon (black arrowhead). $G, H$, Whole-mount ISH with a Dlx2 probe, showing a similar pattern of expression as that seen with $G b x 2$ but in the ventral thalamus. $G$, HH 21; $H$, E 5.5. I, J, Whole-mount ISH for Lunatic fringe, which is expressed at different levels within the anterior synencephalon compared with the posterior synencephalon, indicating that these two regions diverge as development proceeds. I, $\mathrm{HH} \mathrm{20}$; $J$, HH 23.
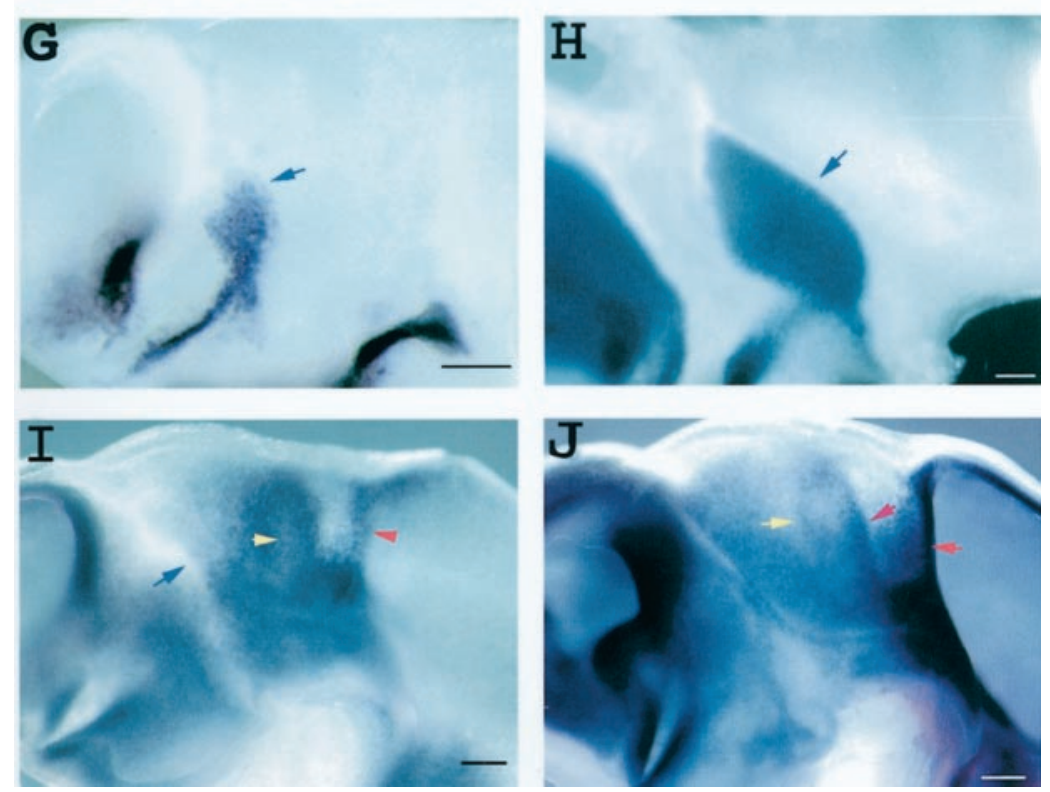

ilarly expressed in the diencephalon. We also examined the expression of NrCAM. At HH 19, CSPG is found in the zli and in the $\mathrm{m} / \mathrm{s}$ boundary as well as in the ventral region of the basal plate (Fig. 5A). At this stage, CSPG is detected throughout the entire dorsoventral extent of the zli, although ventrally this domain is wider anteroposteriorly than the zli. At the $\mathrm{m} / \mathrm{s}$ boundary, CSPG is detected in a broad area extending into the midbrain. At the s/p boundary, CSPG is detected only at the extreme dorsal aspect. This pattern of staining intensifies and sharpens as development proceeds, and by $\mathrm{HH} 22$ (Fig. 5B) strong staining remains within the zli and the $\mathrm{m} / \mathrm{s}$ boundary. However, CSPG is no longer detected in the s/p boundary. Patchy expression of CSPG is also apparent in the ventral aspect of the posterior commissure (Fig. 5B, black arrow) as well as the extreme ventral aspect of the dorsal thalamus. Horizontal sections show that CSPG staining is present 

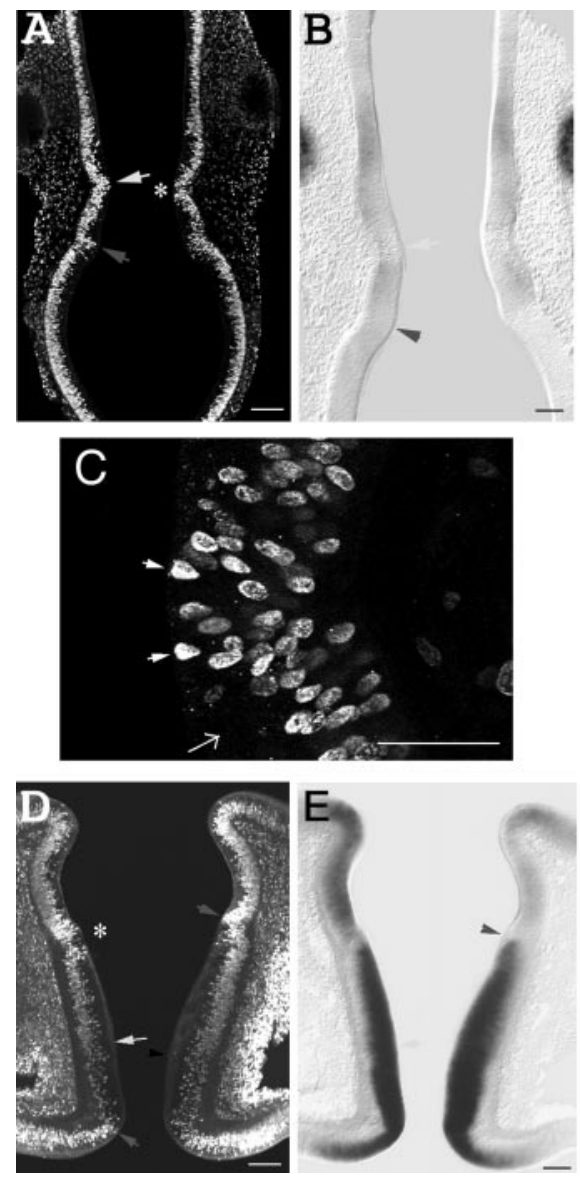
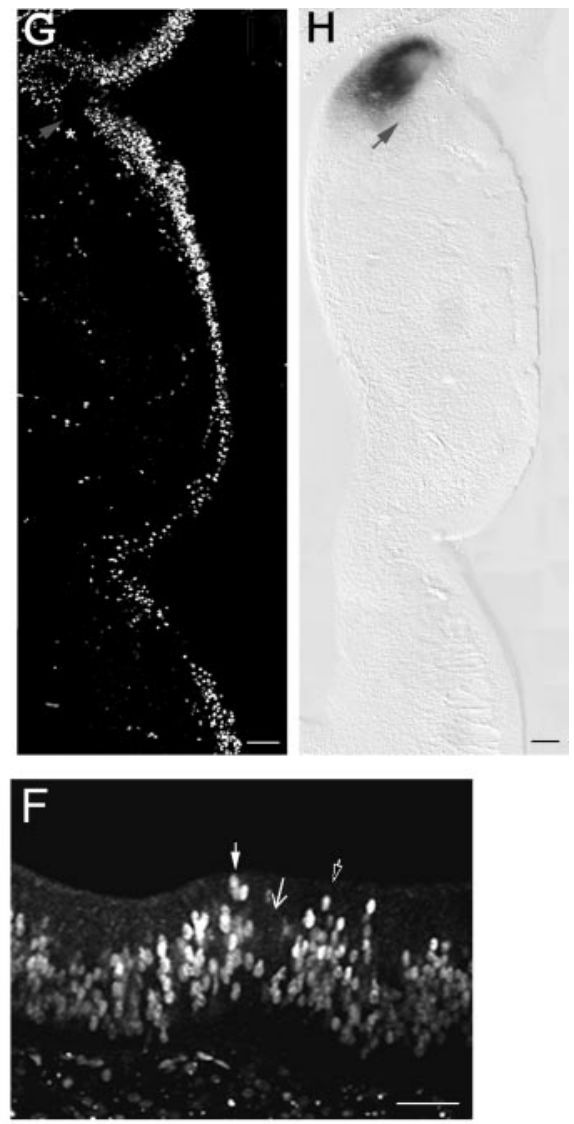

Figure 4. BrdU labeling of S-phase cells. Anterior is at the top and posterior at the bottom, unless stated otherwise. Scale bars, $100 \mu \mathrm{m}$. $A-C$, Horizontal sections of the same BrdU-labeled HH 16 embryo analyzed for Pax6 expression, showing the apical location of S-phase cells at the $\mathrm{m} / \mathrm{s}(A$, gray arrow $)$ and $\mathrm{s} / \mathrm{p}$ ( $A$, white arrow) boundaries. $A$, Confocal image. $B$, Nomarski image. $C$, High magnification of the area labeled with an asterisk in $A$. In $C$, the white arrowheads marks S-phase cells at the ventricular surface within the boundary, and the arrow marks S-phase cells at their normal position on the ventricular-pial axis of the neuroepithelium. $D-F$, Horizontal sections of the same BrdU-labeled HH 21 embryo analyzed with an $L-f n g$ probe, showing the location of apically situated S-phase cells in the zli. At this stage, $\mathrm{S}$-phase cells at the s/p boundary $(D$, white arrow) are situated more basally in the neuroepithelium. $D$, Confocal image. $E$, Nomarski image. $F$, High magnification of the area labeled with asterisk in $D$ (arrow marks the zli). In $F$, the white arrow marks the apically located S-phase cells within the zli, the closed arrow marks an apically located S-phase cell caudal to the zli, and the open arrow marks an adjacent area where there are few BrdU-labeled cells. $G-H$, Horizontal sections of the same BrdU-labeled HH 26 embryo analyzed with a $D l x 2$ probe. $G$, Confocal image; the area without BrdU-labeled cells (asterisk and arrow) is the zli. $H$, Nomarski image. throughout the marginal and mantle zones (Fig. 5C), as well as the ventricular zone of the zli and the $\mathrm{m} / \mathrm{s}$ boundary.

NrCAM is a member of the NCAM family, expressed widely in the CNS (Stoeckli et al., 1997). Low levels of expression are detected throughout the diencephalon at early developmental stages (data not shown). At HH 19, NrCAM is expressed strongly in the zli (Fig. 5D) and within the synencephalon, whereas the boundaries on either side of the synencephalon remain unstained. NrCAM immunoreactivity is also detected in the basal plate. By $\mathrm{HH} 22, \mathrm{NrCAM}$ immunostaining is downregulated in the anterior synencephalon (Fig. $5 E$ ) but is stronger in the zli, in the posterior synencephalon, (Fig. 5E, black arrow), and within the ventral basal plate. NrCAM is present within the marginal zone of the dorsal thalamus and the two synencephalic domains (Fig. $5 F)$ and in the ventricular zone within the zli and the posterior commissure (Fig. 5F, black arrow).

Vimentin, an intermediate filament protein, is among the earliest markers for radial glial cells in the chick CNS (Tapscott et al., 1981). Vimentin staining is faint throughout the diencephalon as early as $\mathrm{HH} 14$ (data not shown), but by HH 16 stronger staining is seen at the boundaries flanking the synencephalon (Fig. 5G). Both domains of vimentin immunostaining are broad and extend into the tissue adjacent to the boundaries. At $\mathrm{HH} 20$, vimentin staining is increased in the $\mathrm{m} / \mathrm{s}$ boundary, the zli, and the basal plate (Fig. $5 H$ ) but is lost from the s/p boundary.

Tenascin, an extracellular matrix glycoprotein (ChiquetEhrismann et al., 1986), is first detected in the $\mathrm{m} / \mathrm{s}$ boundary at HH 18 (Fig. 5I). By HH 22 (Fig. 5J), weak staining for tenascin is also seen in the zli, with stronger staining in the posterior synencephalon and the $\mathrm{m} / \mathrm{s}$ boundary.

Our data reveal that the boundaries within the diencephalon exhibit neither a uniform repertoire of the markers examined nor a uniformly ordered sequence of their expression, such as seen in the rhombomere boundaries. Within the zli, disruption of interkinetic nuclear migration follows the expression of extracellular molecules, whereas these events occur simultaneously at the $\mathrm{m} / \mathrm{s}$ boundary and transiently between $\mathrm{HH} 16$ and 18 at the s/p boundary. Cells within the putative intrasynencephalic boundary do not exhibit a particular phenotype at any stage examined. The different boundary phenotypes are summarized in Table 1.

\section{Not all diencephalic neuromeres are cell lineage restricted}

It has been proposed that some of the phenotypes exhibited by rhombomere boundaries are generated by compartment formation (Lumsden, 1999), which suggests that not all diencephalic boundaries restrict the mixing of cell lineages. To determine whether the subdivisions of the diencephalon represent cell lineage restriction units, we labeled one or a few contiguous cells and later examined their distribution in conjunction with ISH for domain markers. The ventral thalamus was identified by $D l \times 2$, the dorsal thalamus by $G b x 2$, and the posterior synencephalon by Prox.

Clones always demarcate the boundary between the midbrain and the diencephalon (Fig. 6A). Mother cells and their clonal descendants generated from injections between $\mathrm{HH} 10$ and $\mathrm{HH}$ 

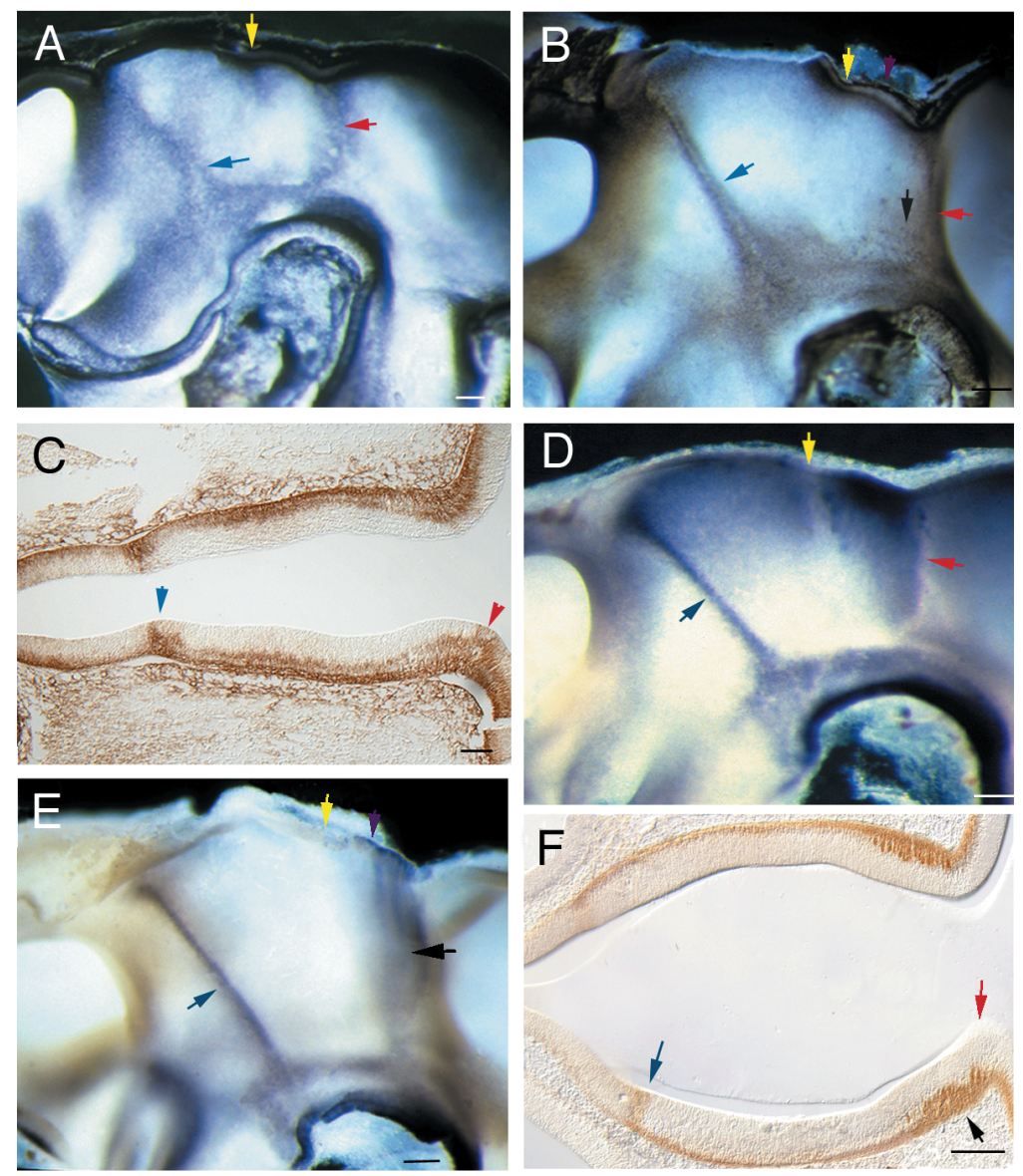
and tenascin. Anterior is to the left unless stated otherwise. Scale bars, $200 \mu \mathrm{m}$. The red arrow indicates the boundary between the midbrain and synencephalon $(\mathrm{m} / \mathrm{s})$. The purple arrow indicates the interface between the anterior and posterior synencephalon. The yellow arrow indicates the boundary between the synencephalon and the parencephalon $(\mathrm{s} / \mathrm{p})$, and the blue arrow marks the intraparencephalic boundary (zli). $A, B$, Wholemount immunohistochemistry with an anti-CSPG antibody, showing expression in the zli and $\mathrm{m} / \mathrm{s}$ boundary only. Embryos have been hemisected and photographed from the ventricular surface. $A, \mathrm{HH} 19 ; B, \mathrm{HH} 22$. $C$, Horizontal vibratome section stained with anti-CSPG showing that CSPG is expressed throughout the diencephalon but is present in the ventricular layer only at the $\mathrm{m} / \mathrm{s}$ boundary and the zli. $D, E$, Whole-mount immunohistochemistry with an anti-NrCAM antibody. Both embryos have been hemisected and photographed from the ventricular surface. Strong expression is seen in the pretectum (black arrow) but is absent from all boundaries except the zli. $D$, HH 19; E, HH 22. F, Horizontal vibratome section, showing the expression of $\mathrm{NrCAM}$ throughout the diencephalon and the ventricular layer expression that is restricted to the zli. $G, H$, Whole-mount immunohistochemistry with an anti-vimentin antibody. Both embryos have been hemisected and photographed from the ventricular surface. Expression at the $\mathrm{s} / \mathrm{p}$ boundary is transient (between $\mathrm{HH} 16$ and $\mathrm{HH} 18$ ) but is more enduring at the $\mathrm{m} / \mathrm{s}$ boundary and the zli. $G$, HH $16 ; H, \mathrm{HH} 20 . I, J$, Whole-mount immunohistochemistry with an anti-tenascin antibody. Tenascin is expressed in the zli and the $\mathrm{m} / \mathrm{s}$ boundary only, but later than the above proteins. Both embryos have been hemisected and photographed from the ventricular surface. $I$, HH 18; $J$, HH 22.
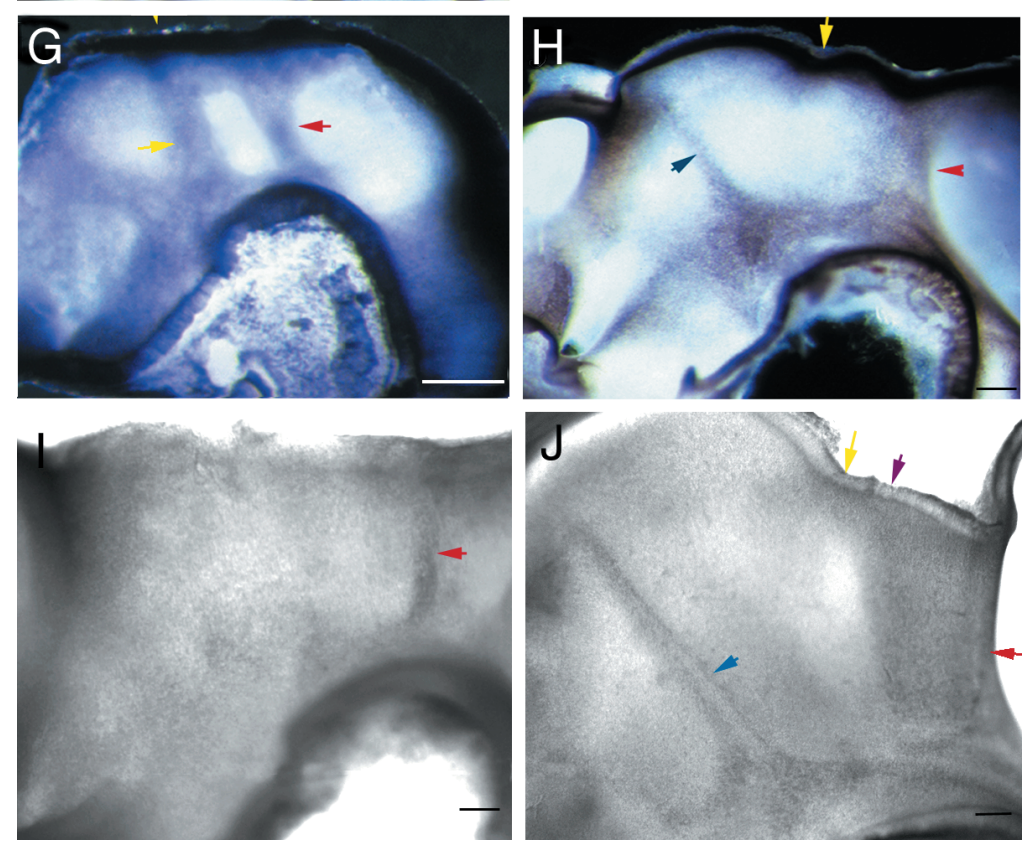

18 were not restricted from crossing the intrasynencephalic boundary (Fig. 6B,C). Labeling at $\mathrm{HH} 18$ produced clones that preferentially stayed together and contained fewer cells compared with injections at earlier stages. Surprisingly, cell lineage restriction was not observed at the $\mathrm{s} / \mathrm{p}$ boundary at any stage of mother cell labeling between $\mathrm{HH} 10$ and $\mathrm{HH} 18$ (Figs. 6D, H). Similar to the situation described above, clones from $\mathrm{HH} 10$ injections were large and dispersed, whereas those at $\mathrm{HH} 18$ remained small, with minimal dispersal of individual progeny.

We have shown previously that the zli originates from a broad compartment, characterized by the absence of $L-f n g$ (Zeltser et al., 2001). Thus, cells labeled in the anterior diencephalon between $\mathrm{HH} 11$ and $\mathrm{HH} 17$ were restricted to the ridge or aligned along the posterior boundary within the dorsal thalamus (Figs. 


\begin{tabular}{|c|c|c|c|c|}
\hline Boundary & $\begin{array}{l}\text { Midbrain- } \\
\text { synencephalic }\end{array}$ & Intrasynencephalic & $\begin{array}{l}\text { Synencephalic/ } \\
\text { parencephalic }\end{array}$ & Zli \\
\hline Morphological ridge & + & $(+)$ & + & $+^{a}$ \\
\hline Increased extracellular spaces & + & & & + \\
\hline Disrupted interkinetic movement & + & & + & $\sim+^{a}$ \\
\hline CSPG & + & & $+{ }^{b}$ & + \\
\hline Tenascin & + & & & + \\
\hline Vimentin & + & & $+^{b}$ & + \\
\hline $\mathrm{NrCam}$ & + & & & + \\
\hline
\end{tabular}

${ }^{a}$ Character appears first ventrally and gradually moves dorsally.

${ }^{b}$ Character seen only at the dorsal aspect of the boundary.

$6 I, J)$. As with labeling in other regions of the diencephalon at $\mathrm{HH} 18$, clones were small and did not disperse widely within the neuroepithelium. The descendants of cells labeled at HH 11 also behaved characteristically, in that they tended to disperse widely within the neuroepithelium and mingle with unlabeled cells.

Clones from labeling injections aimed at the ventral thalamus did not delineate the anterior boundary of the zli (Fig. 6K,L). Rather, they migrated dorsally and in most cases entered the telencephalic vesicle, even when injections were applied ventrally. This pattern of cell movement was also observed when a focal injection of $\mathrm{DiO}$ was applied at $\mathrm{HH} 11$ into the ventral aspect of the ventral thalamus (Fig. $6 K$ ). Therefore, in contrast to the situation in the hindbrain and in contradiction of previous analyses of the diencephalon (Figdor and Stern 1993), not all boundaries in the diencephalon restrict cell movement. The results of the cell lineage analysis are summarized in Table 2.

\section{DISCUSSION}

On the basis of analyses of morphology, molecular markers, and boundary characteristics, we find that the alar plate of the diencephalon is progressively subdivided to form five domains: the ventral and dorsal thalamus, the zli, and the anterior and posterior synencephalon (Fig. 7). These form three distinct regions in the adult: the anterior and posterior synencephalon become the pretectum, the dorsal thalamus is the anlage of the thalamus, and the ventral thalamus forms a set of nuclei associated with thalamocortical communication. The zli, however, appears to have no adult representation other than as the pathway of the mammillothalamic tract.

At HH 13-14, borders of $L$-fng expression delineate the compartment boundaries of the presumptive zli (Zeltser et al., 2001). The first morphological subdivision is at HH 16, when the synencephalon becomes distinct from the parencephalon by adopting neuromeric morphology and expressing Prox. The ridge of the zli, which subdivides the parencephalon into dorsal thalamus posteriorly and ventral thalamus anteriorly, forms gradually between $\mathrm{HH} 19$ and $\mathrm{HH}$ 26. During this period, the dorsal thalamus bulges into the ventricular lumen and expresses $G b x 2$. The ventral thalamus, which expresses $D l x 2$, does not have neuromeric morphology. At $\mathrm{HH} 22$, the posterior synencephalon diverges from the anterior in morphology and the expression of $L-f n g$. Although this last subdivision is not associated with boundary formation, the other subdivisions are linked with the development of specialized boundaries, where cells adopt a specific phenotype. These include ridge formation, disruption of interkinetic movement, and the local expression of extracellular and intracellular molecules. Each of the emerging diencephalic subdivisions man- ifests a unique sequence of morphological and molecular events, reflecting differences in patterning between successive domains, rather than the similarities expected of a segmental series.

A similar pattern of neurogenesis is observed within odd as compared with even rhombomeres at HH 11-16 (Lumsden and Keynes, 1989). In the diencephalon, however, there is no obvious repeat pattern to neurogenesis. Rather, there is an overall ventral to dorsal progression (Bergquist and Kallen, 1954; Keyser, 1972), reflected in the expression of the transcription factors $D l \times 2, G b x 2$, and Prox in the ventral thalamus, dorsal thalamus, and synencephalon, respectively. However, neurogenesis begins earlier in the synencephalon, and Prox expression precedes that of Gbx2 and $D l \times 2$ by three stages. Although $G b \times 2$ has been directly linked to the specification of the dorsal thalamus (Miyashita-Lin et al., 1999), Dlx2 and Prox mouse mutants were less informative (Qiu et al., 1995; Wigle et al., 1999). However, Prox has been linked with the domain-specific regulation of stem cell differentiation (Torii et al., 1999), and its distinct expression within the synencephalon therefore may reflect early specification. Together with the earlier onset of neurogenesis, this would suggest that the synencephalon might be specified independently and before the rest of the diencephalon.

Formation of rhombomeres is associated with the acquisition of specific boundary phenotypes: cell lineage restriction and compartment definition is followed by the differentiation of specialized cells at the compartment interfaces, which are characterized by disruption of interkinetic nuclear migration, ventricular ridge formation, and the expression of cell adhesion and extracellular matrix molecules. Rhombomere boundaries exhibit a high degree of uniformity in their markers and in the sequence in which these are expressed. At all diencephalic domain boundaries identified, disruption of interkinetic nuclear migration is concurrent with ridge formation. However, diencephalic boundaries vary in respect to the rhombomere boundary markers that they express and in the sequence in which these appear. Because the functions of these molecules appear to differ with developmental context (Faissner and Steindler, 1995; Chiquet-Ehrismann et al., 1996; Stoeckli et al., 1997), it would be premature to speculate on their function in diencephalic boundaries.

The absence of NrCAM, a putative axonal guidance molecule (Volkmer et al., 1996), at the mesencephalic/synencephalic boundary is consistent with a function in separating the dorsoventral mesencephalic tracts and the posterior commissure. Cells were never seen to cross from the synencephalon into the midbrain or vice versa, indicating that there is also effective separation of the respective cell populations at this boundary. 

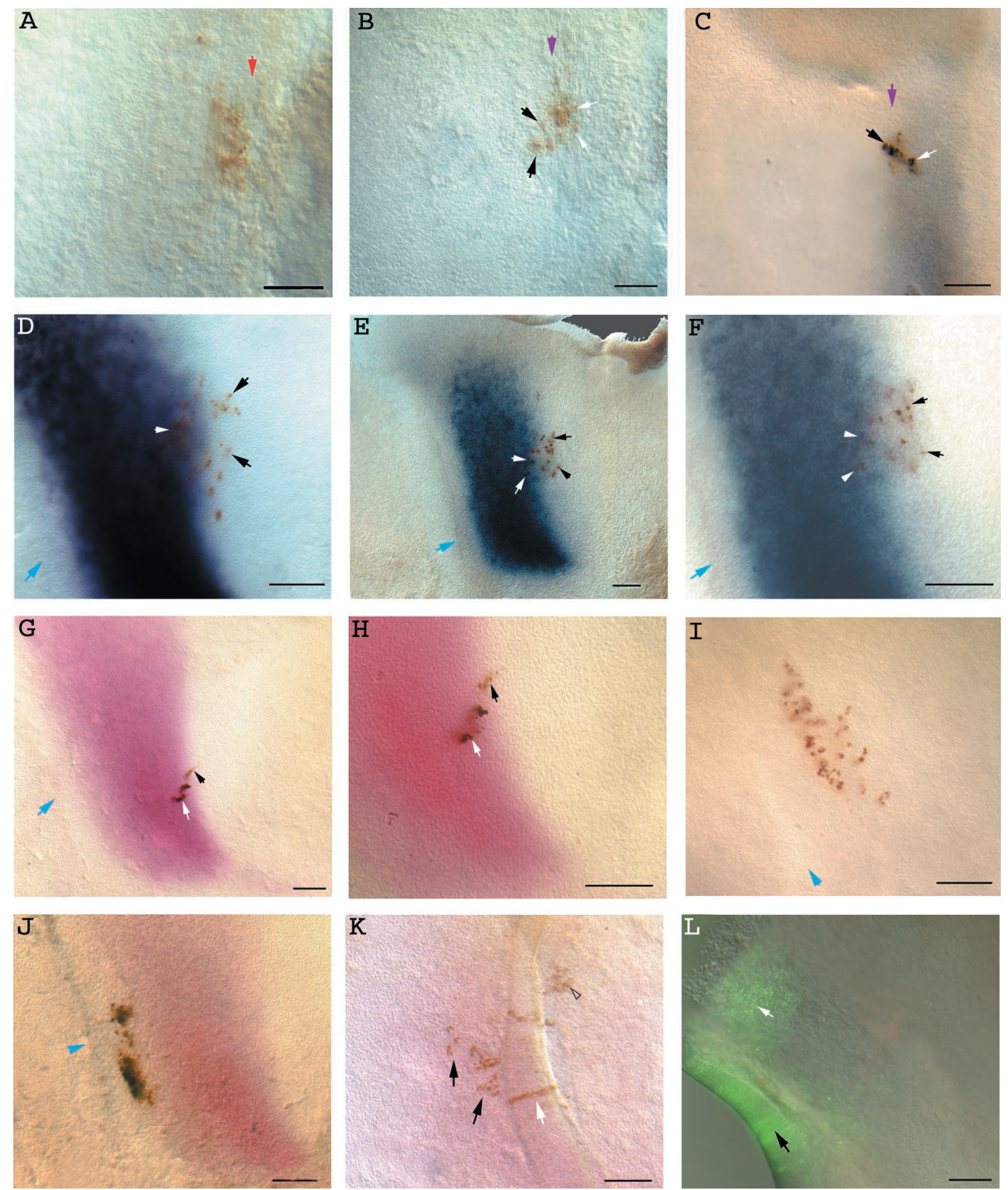

Figure 6. Cell lineage restriction analysis between domains within the synencephalon. Anterior is to the left unless stated otherwise. Scale bars, 100 $\mu \mathrm{m}$. The red arrowhead indicates the boundary between the midbrain and synencephalon $(\mathrm{m} / \mathrm{s})$. The purple arrowhead indicates the interface between the anterior and posterior synencephalon. The blue arrowhead marks the intraparencephalic boundary (zli). All cell labelings were made close to each of the putative boundaries. Embryos have been hemisected and are viewed from the ventricular surface. Dextran-labeled clones have been detected by PO-conjugated streptavidin and a DAB reaction (brown stain). A, Example of a clone respecting the $\mathrm{m} / \mathrm{s}$ boundary; embryo was labeled at $\mathrm{HH} 15$. B, $C$, Examples of clonal spread across the intrasynencephalic boundary. Embryos have been hybridized with a Prox probe to demarcate the posterior synencephalon. Black arrowheads mark part of the clone within the anterior synencephalon, whereas white arrowheads mark part of the clone within the posterior synencephalon. B, Labeled at $\mathrm{HH} 11$ and harvested after $48 \mathrm{hr}$ at $\mathrm{HH} 21$. C, Labeled at $\mathrm{HH} 18$ and harvested after $48 \mathrm{hr}$ at $\mathrm{HH} 25$. $D-H$, Examples of clonal spread between the synencephalon and dorsal thalamus. Embryos have been hybridized with a $G b x 2$ probe to mark the limits of the dorsal thalamus. Black arrowheads mark part of the clone within the synencephalon, whereas white arrowheads mark part of the clone within the dorsal thalamus. $D$, Labeled at $\mathrm{HH} 10$ and harvested $48 \mathrm{hr}$ later at HH 23. $E, F$, Labeled at HH 14 and harvested $48 \mathrm{hr}$ later; $F$ is a higher magnification of E. G, H, Labeled at HH 18 and harvested after $48 \mathrm{hr}$ at HH 25. I, J, Cells labeled in the dorsal thalamus form clones that demarcate the posterior border of the zli. I, Labeled at HH 11 and harvested $48 \mathrm{hr}$ later at $\mathrm{HH} 21$. J, Labeled at HH 17 and harvested $48 \mathrm{hr}$ later at $\mathrm{HH} 25 . K, L$, Cells labeled in the ventral thalamus form clones that disperse into the telencephalic vesicle, showing the lack of clonal restriction between the diencephalon and the secondary prosencephalon. Cells were labeled in the ventral region of the ventral thalamus, and the resulting clones moved dorsally into the telencephalic vesicle. K, Labeled at $\mathrm{HH} 16$ and harvested $48 \mathrm{hr}$ later at $\mathrm{HH} 24$. Black arrows mark clones within the telencephalic vesicle. White arrow marks a clone spanning the ventricular surface at the interface between the telencephalic vesicle and the ventral thalamus. Open arrowhead indicates cells within the ventral thalamus. $\mathrm{H}$, Focal injection with $\mathrm{DiO}$, labeled at $\mathrm{HH}$ 14, and harvested $48 \mathrm{hr}$ later at $\mathrm{HH} 24$. The fluorescence image is overlaid by a Nomarski image. Black arrow shows the part of the clone within the telencephalic vesicle, whereas the white arrow marks the cells that have remained within the ventral thalamus. 


\begin{tabular}{llllll}
\hline \multicolumn{2}{c}{ Table 2. Cell lineage restriction at diencephalic boundaries } & & & \\
& $\begin{array}{l}\text { Midbrain- } \\
\text { synencephalic }\end{array}$ & $\begin{array}{l}\text { Intra- } \\
\text { synencephalic }\end{array}$ & $\begin{array}{l}\text { Synencephalic/ } \\
\text { parencephalic }\end{array}$ & Zli & $\begin{array}{l}\text { Diencephalic/ } \\
\text { telencephalic }\end{array}$ \\
\hline $\begin{array}{l}\text { Boundary } \\
\begin{array}{l}\text { Total number of clones } \\
\text { Number of clones that } \\
\text { crossed the boundary }\end{array}\end{array}$ & 54 & 634 & 353 & 74 & 89 \\
$\begin{array}{c}\text { Number of clones that } \\
\text { respected the boundary }\end{array}$ & 8 & 31 & 42 & 0 & 61 \\
$\begin{array}{c}\text { Number of uninformative } \\
\text { clones }\end{array}$ & 46 & 0 & 0 & 74 & 0 \\
\hline
\end{tabular}

Each embryo was injected once, and in each injection between one and three cells were labeled. Injections were targeted randomly to the area around each presumptive boundary; therefore, clones were often too far from the boundary to be informative. The zli refers to clones labeled in the caudal region of the parencephalon.

We did not identify any boundary-specific characteristics between the anterior and posterior synencephalon, the distinction between which can be made only by morphology. However, the change in morphology and expression of $L-F n g$, at approximately $\mathrm{HH} 22$, suggests that the two regions eventually diverge. By contrast with Figdor and Stern (1993), we find that cell movement between the two domains is not restricted, even when the cells were labeled as late as $\mathrm{HH} 20$, some time after the segmentation of the synencephalon proposed by these authors. Because of the high degree of vascularization at later stages, it was not possible to extend the analysis to $\mathrm{HH} 22$, when the change in morphology occurs. However, if an intrasynencephalic boundary is formed at this later stage, it would be contemporaneous with the disappearance of some of the other diencephalic boundaries.

Ridge formation and apical localization of S-phase cells are transient at the synencephalic/parencephalic boundary, between $\mathrm{HH} 16$ and $\mathrm{HH}$ 18, and there is no cell lineage restriction. However, the disappearance of this boundary is not associated with a widespread dispersal of cells between the two domains, perhaps because cell dispersal after $\mathrm{HH} 18-19$ is much reduced compared with earlier stages.

Unlike the other diencephalic boundaries, the zli is not a border between two cellular domains but is itself a narrow compartment with cell lineage-restricted boundaries both anteriorly with ventral thalamus and posteriorly with dorsal thalamus. These boundaries colocalize with borders of $L$-fng expression at $\mathrm{HH}$ 13-14 (Zeltser et al., 2001). The compartment initially constitutes approximately one-third of the forebrain vesicle and later narrows to form the definitive zli. Formation of the ridge and the disruption of interkinetic movements between $\mathrm{HH} 19$ and $\mathrm{HH}$ 26 follow the ventral to dorsal expansion of the expression of Gbx2 and Dlx2. In contrast, CSPG, vimentin, and NrCAM are expressed at HH 20 throughout the dorsoventral extent of the zli. The significance of the delay in the acquisition of a boundary phenotype relative to cell lineage restriction is not known.

As for the intrasynencephalic subdivision, we did not identify a ridge or boundary phenotype between the ventral thalamus and the secondary prosencephalon. Furthermore, clonal or polyclonal descendents of cells labeled in the ventral thalamus spread anteriorly into the telencephalic vesicle at all stages tested. This contrasts with the descendants of cells labeled in the anterior part of the dorsal thalamus, which consistently align along the boundary with the zli. Therefore, our cell labeling studies demonstrate that the broad anterior spread of polyclones in the ventral thalamus differs from the general ventral to dorsal dispersal seen in the rest of the diencephalon. In light of the extensive cell mixing between the two domains, it is not clear how the ventral thalamus is specified separately from the secondary prosencephalon. Unlike the other diencephalic domains, the ventral thalamus is not competent to express midbrain markers (Crossley et al., 1996), suggesting that ventral thalamus patterning is more closely associated with the secondary prosencephalon and may even be considered a part of it. In this view, the zli would represent a pivotal structure along the neuraxis, segregating a secondary (enlarged) prosencephalon from the remainder of the neural tube. This distinction in turn may have its origins in the distinct inductive processes responsible for the early specification of the prechordal neural plate, overlying the prechordal mesendoderm, versus the epichordal neural plate, which overlies notochord.

CNS development in both vertebrates and invertebrates is associated with the formation of neuromeres. The essence of neuromery, as for the segmentation of other systems such as the mesodermal somites, is the formation of a metameric (i.e., repetitive) series of modules that are morphologically similar to one another and share a common ground plan. The classic definition of neuromery includes the presumption that "the cells of one neuromere do not extend into another neuromere" (Orr, 1887), a notion that presaged the more modern concepts of lineage restriction and compartition that have become a principal criterion for neuromery.

The diencephalon can be subdivided into five domains on the basis of distinct morphological, cellular, and molecular criteria (Fig. 7), but evidence for reiteration through these domains of any feature, whether morphological or molecular, is scant. Furthermore, only the synencephalon has the classic neuromeric morphology of a trough delineated by ridges (Orr, 1887), and only the zli is truly a compartment defined by cell lineage restriction. The lack of an overtly reiterated pattern as assessed by our morphological, cellular, and molecular criteria, the lack of compartition (except for the zli), and the lack of uniformity in the expression of boundary markers suggest that the diencephalon should not be regarded as being a truly segmented region of the neuraxis, as has been suggested previously (Puelles et al., 1987; Figdor and Stern, 1993). Rather, our data substantiate the view held by many of the early workers in the field (Orr, 1887; Streeter, 1933), who saw no evidence at the morphological level for segmentation in CNS regions other than the hindbrain. The five domains of the avian diencephalon are likely to be specified independently of one another. 


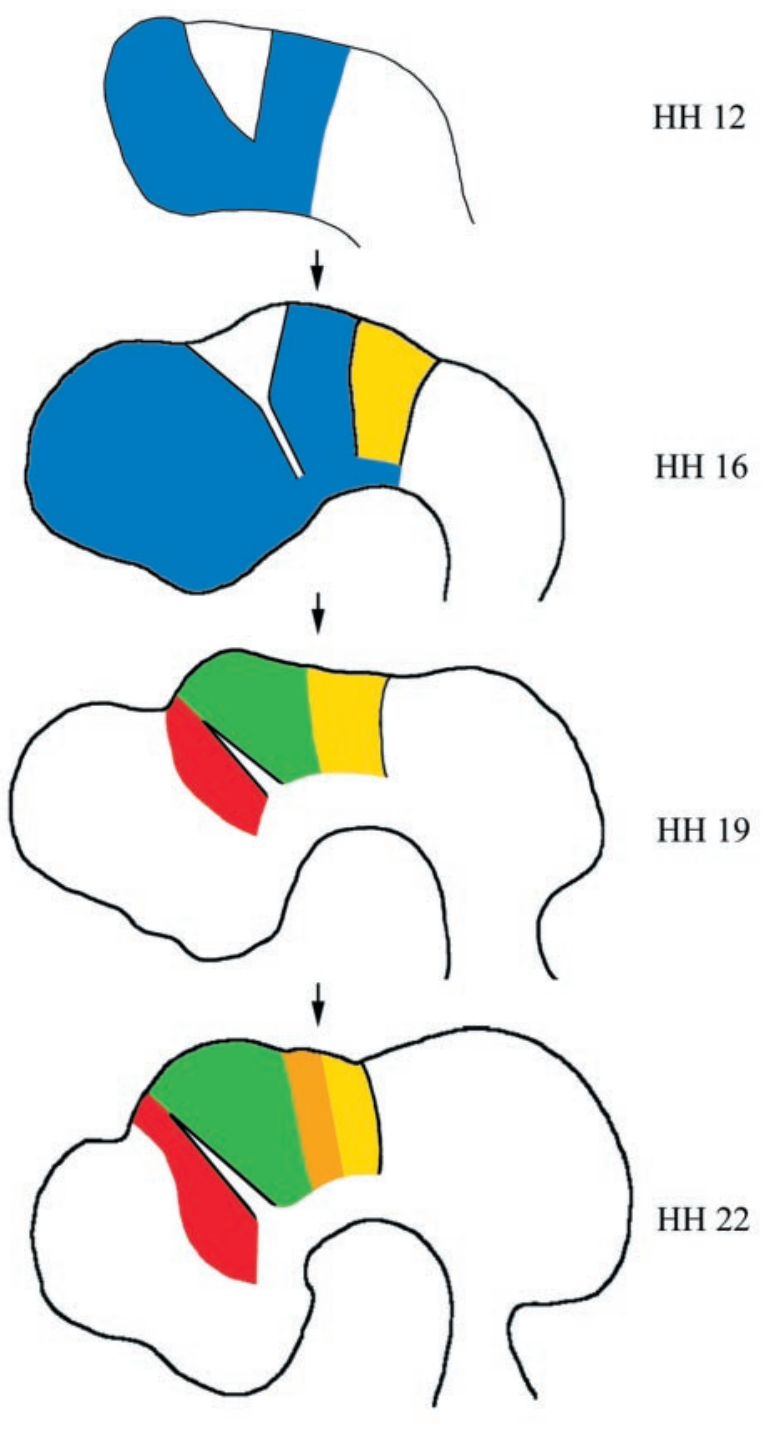

Parencephalon + Secondary Prosencephalon Synencephalon

Dorsal Thalamus Ventral Thalamus Ant. Synencephalon

Figure 7. Diagrammatic summary of diencephalic subdivision. The progressive appearance of the five diencephalic subdivisions is shown at a series of Hamburger and Hamilton stages of chick development. Boundaries that exhibit lineage restriction $(\mathrm{m} / \mathrm{s}$, anterior and posterior zli boundaries, and the transient $\mathrm{s} / \mathrm{p}$ boundary) are shown as black lines.

\section{REFERENCES}

Bergquist H, Kallen B (1954) Notes on the early histogenesis and morphogenesis of the central nervous system in vertebrates. J Comp Neurol 100:627-659.

Bruckner K, Perez L, Clausen H, Cohen S (2000) Glycosyltransferase activity of fringe modulates Notch-Delta interactions. Nature 406:411-415.

Bulfone A, Puelles L, Porteus MH, Frohman MA, Martin GR, Rubenstein JL (1993) Spatially restricted expression of Dlx-1, Dlx-2 (Tes-1), Gbx-2, and Wnt-3 in the embryonic day 12.5 mouse forebrain defines potential transverse and longitudinal segmental boundaries. J Neurosci 13:3155-3172.

Cambronero F, Puelles L (2000) Rostrocaudal nuclear relationships in the avian medulla oblongata: a fate map with quail chick chimeras. J Comp Neurol 427:522-545.
Chiquet-Ehrismann R, Mackie EJ, Pearson CA, Sakakura T (1986) Tenascin: an extracellular matrix protein involved in tissue interactions during fetal development and oncogenesis. Cell 47:131-139.

Coggeshall RE (1964) A study of diencephalic development in the albino rat. J Comp Neurol 122:241-270.

Crossley PH, Martinez S, Martin GR (1996) Midbrain development induced by FGF8 in the chick embryo. Nature 380:66-68.

Faissner A, Steindler D (1995) Boundaries and inhibitory molecules in developing neural tissues. Glia 13:233-254.

Figdor MC, Stern CD (1993) Segmental organization of embryonic diencephalon. Nature 363:630-634.

Fleming RJ, Gu Y, Hukriede NA (1997) Serrate-mediated activation of Notch is specifically blocked by the product of the gene fringe in the dorsal compartment of the Drosophila wing imaginal disc. Development 124:2973-2981.

Fraser S, Keynes R, Lumsden A (1990) Segmentation in the chick embryo hindbrain is defined by cell lineage restrictions. Nature 344:431-435.

Grove EA, Tole S, Limon J, Yip L, Ragsdale CW (1998) The hem of the embryonic cerebral cortex is defined by the expression of multiple Wnt genes and is compromised in Gli3-deficient mice. Development 125:2315-2325.

Guthrie S, Butcher M, Lumsden A (1991) Patterns of cell division and interkinetic nuclear migration in the chick embryo hindbrain. J Neurobiol 22:742-754.

Heyman I, Faissner A, Lumsden A (1995) Cell and matrix specializations of rhombomere boundaries. Dev Dyn 204:301-315.

Keyser A (1972) The development of the diencephalon of the Chinese hamster. Acta Anat 83[Suppl 59]:1-178.

Klein T, Arias AM (1998) Interactions among Delta, Serrate and Fringe modulate Notch activity during Drosophila wing development. Development 125:2951-2962.

Lawrence PA, Struhl G (1996) Morphogens, compartments and pattern: lessons from Drosophila? Cell 85:951-961.

Layer PG, Alber R (1990) Patterning of chick brain vesicles as revealed by peanut agglutinin and cholinesterases. Development 109:613-624.

Lumsden A (1999) Closing in on rhombomere boundaries. Nat Cell Biol $1: \mathrm{E} 83-85$.

Lumsden A, Keynes R (1989) Segmental patterns of neuronal development in the chick hindbrain. Nature 337:424-428.

Lumsden A, Krumlauf R (1996) Patterning the vertebrate neuraxis. Science 274:1109-1115.

Mellitzer G, Xu Q, Wilkinson DG (1999) Eph receptors and ephrins restrict cell intermingling and communication. Nature 400:77-81.

Miyashita-Lin EM, Hevner R, Wassarman KM, Martinez S, Rubenstein JL (1999) Early neocortical regionalization in the absence of thalamic innervation. Science 285:906-909.

Moloney DJ, Panin VM, Johnston SH, Chen J, Shao L, Wilson R, Wang Y, Stanley P, Irvine KD, Haltiwanger RS, Vogt TF (2000) Fringe is a glycosyltransferase that modifies Notch. Nature 406:369-375.

Oliver G, SosaPineda B, Geisendorf S, Spana EP, Doe CQ, Gruss P (1993) Prox 1, a prospero-related homeobox gene expressed during mouse development. Mech Dev 44:3-16.

Orr HA (1887) Contributions to the embryology of the lizard. J Morphol 2:51-96.

Panin VM, Papayannopoulos V, Wilson R, Irvine KD (1997) Fringe modulates Notch-ligand interactions. Nature 387:908-912.

Puelles L, Amat JA, Martinez-de-la-Torre M (1987) Segment-related, mosaic neurogenetic pattern in the forebrain and mesencephalon of early chick embryos: I. Topography of AChE-positive neuroblasts up to stage HH18. J Comp Neurol 266:247-268.

Qiu M, Bulfone A, Martinez S, Meneses JJ, Shimamura K, Pedersen RA, Rubenstein JL (1995) Null mutation of Dlx-2 results in abnormal morphogenesis of proximal first and second branchial arch derivatives and abnormal differentiation in the forebrain. Genes Dev 9:2523-2538.

Redies C, Ast M, Nakagawa S, Takeichi M, Martinez-de-la-Torre M, Puelles L (2000) Morphologic fate of diencephalic prosomeres and their subdivisions revealed by mapping cadherin expression. J Comp Neurol 421:481-514.

Rubenstein JLR, Martinez S, Shimamura K, Puelles L (1994) The embryonic vertebrate forebrain: the prosomeric model. Science 266:578-580

Stoeckli ET, Sonderegger P, Pollerberg GE, Landmesser LT (1997) Interference with axonin-1 and NrCAM interactions unmasks a floorplate activity inhibitory for commissural axons. Neuron 18:209-221.

Streeter GL (1933) The status of metamerism in the central nervous system of chick embryos. J Comp Neurol 57:455-476.

Tapscott SJ, Bennett GS, Holtzer H (1981) Neuronal precursor cells in the chick neural tube express neurofilament proteins. Nature 292:836-838.

Torii M, Matsuzaki F, Osumi N, Kaibuchi K, Nakamura S, Casarosa S, 
Guillemot F, Nakafuku M (1999) Transcription factors Mash-1 and Prox-1 delineate early steps in differentiation of neural stem cells in the developing central nervous system. Development 126:443-456.

Vaage S (1969) The segmentation of the primitive neural tube in chick embryos (Gallus domesticus). A morphological, histochemical and autoradiographical investigation. Erg Anat Entwick 41:3-87.

Volkmer H, Leuschner R, Zacharias U, Rathjen FG (1996) Neurofascin induces neurites by heterophilic interactions with axonal $\mathrm{NrCAM}$ while NrCAM requires F11 on the axonal surface to extend neurites. J Cell Biol 135:1059-1069.

von Baer (1828) Uber die Enwicklungsgeschichte der Thiere, Beobachtung und Reflexion. Borntrager, Konigsberg.
Wigle JT, Chowdhury K, Gruss P, Oliver G (1999) Prox1 function is crucial for mouse lens-fiber elongation. Nat Genet 21:318-322.

Wizenmann A, Lumsden A (1997) Segregation of rhombomere by differential chemoaffinity. Mol Cell. Neurosci 9:448-459.

Xu Q, Mellitzer G, Robinson V, Wilkinson DG (1999) In vivo cell sorting in complementary segmental domains mediated by Eph receptors and ephrins. Nature 399:267-271.

Yoon M, Puelles L, Redies C (2000) Formation of cadherin-expressing brain nuclei in diencephalic alar plate divisions. J Comp Neurol 421: 461-480

Zeltser L, Larsen C, Lumsden A (2001) A novel developmental compartment in the forebrain regulated by Lunatic fringe. Nat Neurosci, in press. 